\title{
Design of Dual-Band Microstrip Rat Race Coupler with Circuit Miniaturization
}

\author{
Ching-Luh Hsu ${ }^{1,2}$, Chin-Wei Chang ${ }^{1}$ and Jen-Tsai Kuo ${ }^{1}$ \\ ${ }^{1}$ Department of Communication Engineering, National Chiao Tung University \\ ${ }^{1} 1001$ Tahsueh Rd., Hsinchu, 300, TAIW AN \\ ${ }^{2}$ Department of Electronics Engineering, Ta Hwa Institute of Technology
}

\begin{abstract}
Rat race ring coupler with stepped-impedance sections is designed to have a dual-band characteristic. This is realized by attaching open stubs to both ends of each steppedimpedance section to create a phase of $90^{\circ}$ at two designated frequencies. Transcendental equations are derived for solving impedance ratio $R$ and lengths of the stepped-impedance sections. Circuit with miniaturized area can be obtained if $R>1$ is chosen. Such microstrip ring hybrids operating at 2.45/5.2 GHz with $R=$ 1 and $R=4$ are designed and fabricated. Experiments are performed to confirm the theory.
\end{abstract}

Index Terms - Directional coupler, dual-band, miniaturization, rat race coupler, stepped-impedance.

\section{INTRODUCTION}

Many mobile and wireless communication systems have been implemented with dual-band operation. For saving cost in system integration, RF components with a dual-band characteristic are of great interest; especially those use a single circuit to operate at the two specified frequencies. Examples include the dual-band low-noise amplifiers (LNA) in [1], the monopole antennas in [2], the parallel-coupled line filters in [3], and the multi-standard RF transceivers in [4].

Recently, dual-band branch-line couplers [5-8] and rat race couplers [9] are reported. In [5], composite right/left-handed transmission lines are used to replace the $\lambda / 4$ arms of a branch-line coupler for $900 / 1800 \mathrm{MHz}$ operation. Additional stubs in [6] and extra cross-coupled branches in [7] are attached to quadrature hybrids for the dual-band purpose. In [8], three-branch-line structure is devised for $2.4 / 5 \mathrm{GHz}$ branch-line couplers with bandwidths of $16 \%$ and $18 \%$ for the lower and upper bands, respectively. In [9], a 900/2000 MHz rat race coupler is devised by adding two short-circuited stubs and by modifying the length and impedance of the branch lines in the conventional structure.

Size reduction is also an important issue for distributed circuits operating at relatively low RF frequencies. Circuit miniaturization of a rat race coupler is of particular interest since the conventional design has a circumference of $6 \lambda / 4$. Probably, the most intuitive way to reduce the area is to use multi-folded traces as in [10] and fractal-line configuration in [11]. Alternatively, by replacing each $\lambda / 4$-section with a stepped-impedance section, the rat race developed in [12] saves more than $75 \%$ of area.
In this paper, a design of rat race coupler incorporating dual-band operation with circuit miniaturization is proposed. Based on the traditional circuit topology, each $\lambda / 4$-section is replaced by a stepped-impedance section with additional open stubs attaching to its ends. Transmission line theory and twoport network analysis are employed for the circuit design. In particular, certain design graphs are devised to solve the transcendental equations for determining the circuit parameters. Two experimental microstrip dual-band rat race hybrids are simulated and measured for demonstration.

\section{DUAL-BAND OPERATION AND CIRCUIT MiNIATURIZATION}

Fig. 1 shows the layout of the proposed circuit. It consists of six unit cells. Each cell consists of two high- $Z$ sections on both sides with two open stubs being attached to their ends and a low- $Z$ section in the middle. In circuit synthesis, equivalence of the unit cell to a quarter-wave section should be established at two designated frequencies. Fig. 2 shows the circuit schematic of a unit cell, where the two admittances $j B(f) / 2$ will be implemented by the shunt stubs. Since the circuit is symmetric about its center, by setting the reference plane $P-P$ ' open- and short-circuited, respectively, the input admittances for even- and odd-mode, $Y_{e}=Y_{11}+Y_{21}$ and $Y_{o}=$ $Y_{11}-Y_{21}$, can be readily derived as follows:

$$
\begin{aligned}
& Y_{11}+Y_{21}=j \frac{B}{2}+j \frac{1}{Z_{1}} \frac{R \tan \theta_{2}+\tan \theta_{1}}{1-R \tan \theta_{1} \tan \theta_{2}} \\
& Y_{11}-Y_{21}=j \frac{B}{2}+j \frac{1}{Z_{1}} \frac{-R \cot \theta_{2}+\tan \theta_{1}}{1+R \tan \theta_{1} \cot \theta_{2}}
\end{aligned}
$$

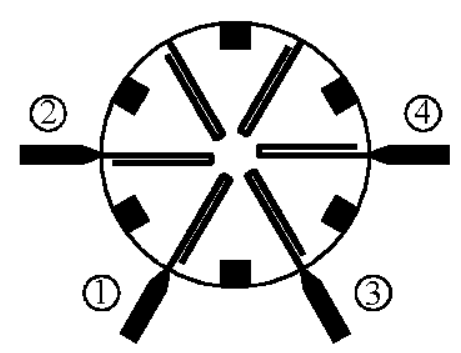

Fig. 1. Layout of the dual-band rat race coupler. 


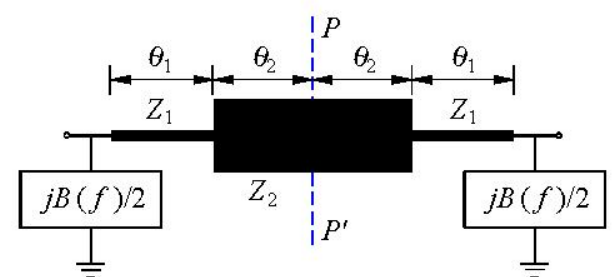

Fig. 2. Schematic of a unit cell of the proposed coupler.

where $Y_{i j}$ denotes the $Y$-parameters of the two-port and $R=$ $Z_{1} / Z_{2}$ is called the impedance ratio of the stepped-impedance line section. On the other hand, the $Y$-matrix of a straight $\lambda / 4$ section with characteristic impedance $Z_{T}$ can be expressed as

$$
Y_{\lambda / 4}=\left[\begin{array}{cc}
0 & j\left(1 / Z_{T}\right) \\
j\left(1 / Z_{T}\right) & 0
\end{array}\right]
$$

It is known that $Z_{T}=\sqrt{2} Z_{0}$ in the traditional $6 \lambda / 4$-ring design, where $Z_{o}$ is the reference port impedance. Assume that the two designated operation frequencies are $f_{1}$ and $f_{2}=n f_{1}$. By enforcing the $Y_{i j}$ entries in (1) and (2) identical to those of (3) at these two frequencies, four equations can be obtained as follows:

$$
\begin{aligned}
& y_{21}\left(f_{1}\right)=\frac{1}{2}\left(\frac{R \tan \theta_{2}+\tan \theta_{1}}{1-R \tan \theta_{1} \tan \theta_{2}}-\frac{-R \cot \theta_{2}+\tan \theta_{1}}{1+R \tan \theta_{1} \cot \theta_{2}}\right) \\
& =\frac{Z_{1}}{Z_{T}} \\
& y_{21}\left(n f_{1}\right)=\frac{1}{2}\left(\frac{R \tan n \theta_{2}+\tan n \theta_{1}}{1-R \tan n \theta_{1} \tan n \theta_{2}}-\frac{-R \cot n \theta_{2}+\tan n \theta_{1}}{1+R \tan n \theta_{1} \cot n \theta_{2}}\right) \\
& =\frac{Z_{1}}{Z_{T}} \\
& B\left(f_{1}\right)=\frac{-1}{Z_{1}}\left(\frac{R \tan \theta_{2}+\tan \theta_{1}}{1-R \tan \theta_{1} \tan \theta_{2}}+\frac{-R \cot \theta_{2}+\tan \theta_{1}}{1+R \tan \theta_{1} \cot \theta_{2}}\right)
\end{aligned}
$$

(6)

$$
B\left(n f_{1}\right)=\frac{-1}{Z_{1}}\left(\frac{R \tan n \theta_{2}+\tan n \theta_{1}}{1-R \tan n \theta_{1} \tan n \theta_{2}}+\frac{-R \cot n \theta_{2}+\tan n \theta_{1}}{1+R \tan n \theta_{1} \cot n \theta_{2}}\right)
$$

where $y_{21}\left(f_{1}\right)=\operatorname{Im}\left[Y_{21}\left(f_{1}\right) \times Z_{1}\right]$ and $y_{21}\left(n f_{1}\right)=\operatorname{Im}\left[Y_{21}\left(n f_{1}\right) \times\right.$ $Z_{1}$ ]. Note that the sign of the shunt admittance $B\left(f_{1}\right)$ is not necessarily the same as that of $B\left(n f_{1}\right)$.

If the impedance ratio $R=1$, the solution to the above equations can be readily obtained and explicitly expressed as

$$
\begin{aligned}
& \theta_{1}+\theta_{2}=\frac{1}{2}\left(\frac{\pi}{1+n}\right) \\
& Z_{1}=Z_{T} \csc \frac{\pi}{1+n}
\end{aligned}
$$

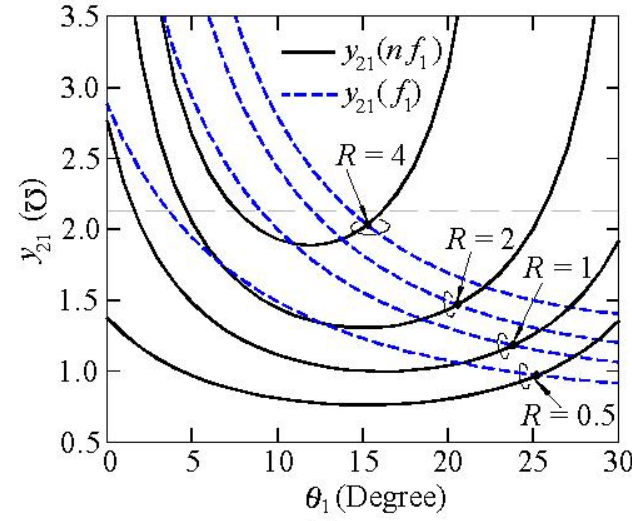

(a)

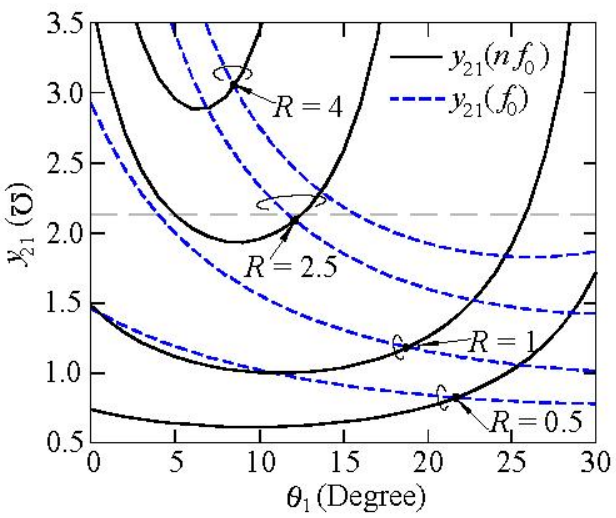

(b)

Fig. 3. Design graphs for determining $\theta_{1}$ and $R$. $y_{21}=2.12$ for the dashed line. (a) $\theta_{2}=5^{\circ}$. (b) $\theta_{2}=10^{\circ}$. Both $\theta_{1}$ and $\theta_{2}$ values are referred to $f_{1}$.

$$
B\left(f_{0}\right)=-B\left(n f_{0}\right)=\frac{2}{Z_{T}} \cos \frac{\pi}{1+n}
$$

When $R \neq 1$, values of $\theta_{1}$ and $\theta_{2}$ can be obtained by enforcing (4) identical to (5). Provided $n=2.12$ (for operation at $5.2 / 2.45 \mathrm{GHz}$ ), Fig. 3(a) and Fig. 3(b) plot both $y_{21}\left(f_{1}\right)$ and $y_{21}\left(n f_{1}\right)$ curves against $\theta_{1}$ for various $R$ values when $\theta_{2}=5^{\circ}$ and $10^{\circ}$, respectively. Both $\theta_{1}$ and $\theta_{2}$ are evaluated at $f_{1}$. For each $R$, the intersection point of the $y_{21}\left(f_{1}\right)$ and $y_{21}\left(n f_{1}\right)$ curves gives the solution. The $Z_{1}$ value can then be obtained by substituting the $y_{21}$ solution into (4) or (5).

Based on the solutions in Fig. 3, circuit miniaturization can be obtained simultaneously if $R>1$ is chosen, since $\theta_{1}$ value decreases when $R$ is increased. At the same time, however, $y_{21}$ $=Z_{1} / Z_{T}$ also increases. By the standard microstrip process, there is an upper limit for realizable $Z_{1}$. This means that the miniaturization cannot be arbitrary. For a microstrip line on a 0.508 -mm substrate with $\varepsilon_{r}=2.2$, the upper limit of $Z_{1}$ is about $150 \Omega$, so that the constraint on $y_{21}$ is

$$
y_{21} \leq \frac{150}{\sqrt{2} Z_{0}}
$$


Given $Z_{0}=50 \Omega, y_{21}$ must be no more than 2.12, as indicated by the horizontal dashed line in Fig. 3. It indicates that $R$ values for $\theta_{2}=5^{\circ}$ and $\theta_{2}=10^{\circ}$ can be chosen up to 4.0 and 2.5, respectively. Obviously, the former offers a design with smaller size.

The last step is to realize the admittances $j B(f) / 2$ in Fig. 2 by shunt open stubs. The admittances of the stubs at $f_{1}$ and $f_{2}$ can be written as

$$
\begin{gathered}
B\left(f_{1}\right)=\frac{1}{Z_{S}} \tan \theta_{S} \\
B\left(n f_{1}\right)=\frac{1}{Z_{S}} \tan n \theta_{S}
\end{gathered}
$$

where $\theta_{S}$ and $Z_{S}$ denote its electrical length and characteristic impedance, respectively. Given $n$, these two equations are used to solve $\theta_{S}$ and $Z_{S}$ based on the values calculated by (6) and (7) after $R$ is properly chosen.

\section{Simulation AND MEAsurEMENT RESUlts}

Table I summarizes the design parameters of the dual-band rat race coupler for $R=1$ and $R=4$. Fig. 4 compares simulation and measured responses of the dual-band rat race coupler with $R=4$. The operating frequencies are 2.45 and $5.2 \mathrm{GHz}$, and the linewidths of the low- $Z$ section, hi- $Z$ section, and the shunt stub are $2.245,0.21$ and $0.444 \mathrm{~mm}$, respectively. The simulation is done by the IE3D [14]. The $\left|S_{11}\right|$ and $\left|S_{21}\right|$ responses are shown in Fig. 4(a), and the $\left|S_{31}\right|$ and $\left|S_{41}\right|$ in Fig. 4(b). The measured return loss and isolation are better than 30 $\mathrm{dB}$ at the two designated frequencies. Detailed measured data show that total power losses of the circuit, $P_{L}=1-\left|S_{11}\right|^{2}-\left|S_{21}\right|^{2}-$ $\left|S_{31}\right|^{2}-\left|S_{41}\right|^{2}$, are $4.3 \%$ and $6.4 \%$ at 2.45 and $5.2 \mathrm{GHz}$, respectively. Fig. 4(c) and 4(d) plot the responses of the phase differences $\angle S_{31}-\angle S_{21}$ and $\angle S_{42}-\angle S_{12}$, respectively. Good agreement between the simulation and measurement results can be observed.

Fig. 5 plots the magnitude responses of $\left|S_{11}\right|,\left|S_{21}\right|,\left|S_{31}\right|$ and $\left|S_{41}\right|$ for $R=1$. It is found that the couplers with $R=1$ and $R=$ 4 have more or less the same bandwidths at the two designated frequencies. Total power losses of the circuit for the case of $R=1$ are $3.3 \%$ and $5.2 \%$ at 2.45 and $5.2 \mathrm{GHz}$, respectively. Compared with the measured data for the above design with $R=4$, it is found that the extra power loss caused by stepped-impedance configuration is about $1 \%$.

In comparison with the area of a conventional 6 $6 \lambda / 4$-ring hybrid operating at $f=f_{1}$, the designed dual-band coupler with $R=1$ uses only $41 \%$ of the area. If $R=4$ is chosen, a further size reduction of $50 \%$ can be obtained. In other words, the size reduction of the dual-band stepped-impedance coupler is better than $79 \%$. The bandwidths of the circuit at the two operation frequencies, however, are much smaller than that of the traditional design.

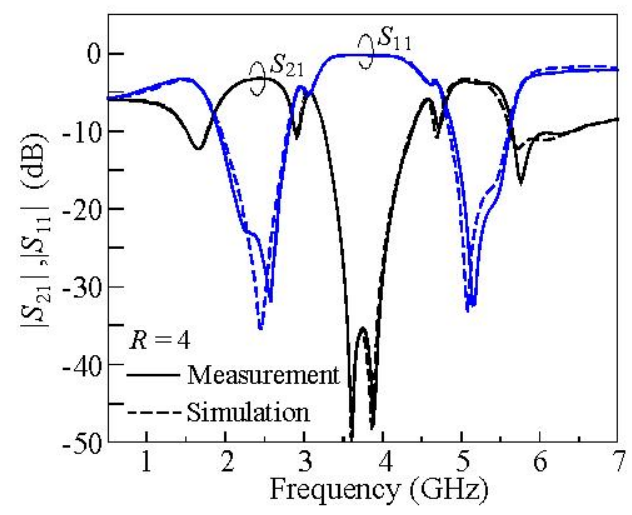

(a)

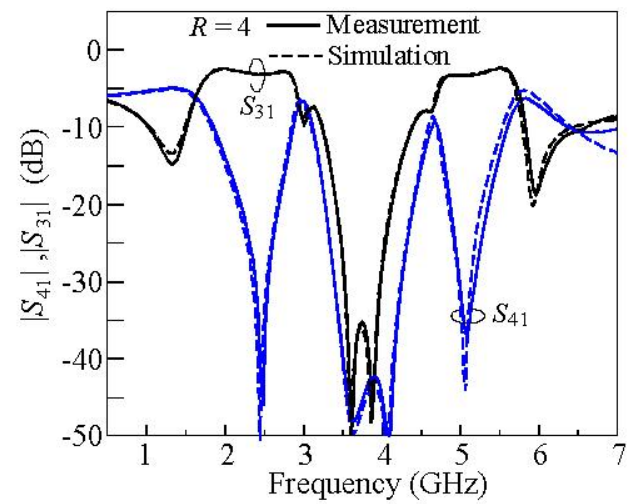

(b)

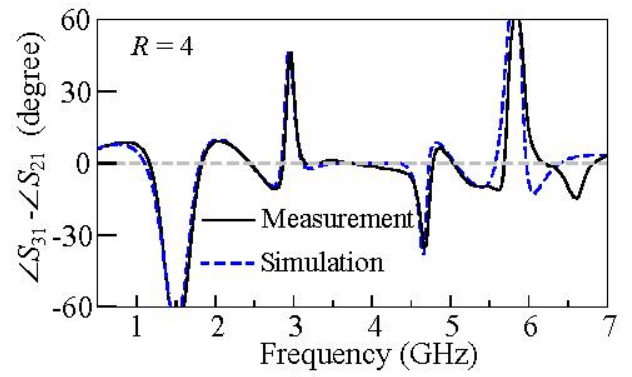

(c)

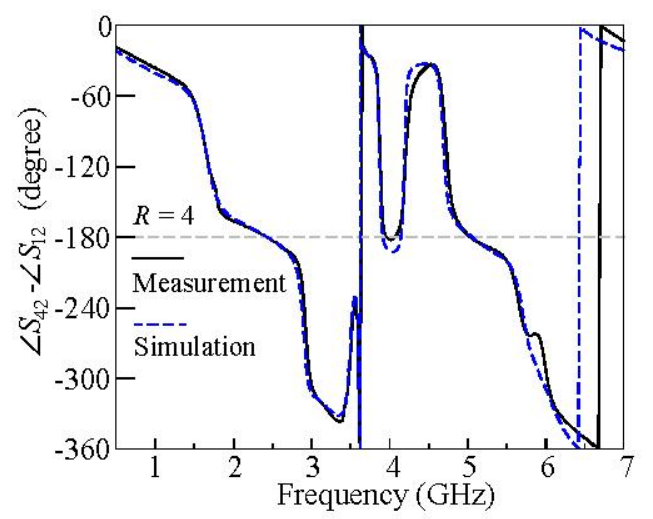

(d)

Fig. 4. Performance of the $2.45 / 5.2 \mathrm{GHz}$ rat race coupler with $R=4$. (a) $\left|S_{11}\right|$ and $\left|S_{21}\right|$. (b) $\left|S_{31}\right|$ and $\left|S_{41}\right|$. (c) $\angle S_{31}-\angle S_{21}$. (d) $\angle S_{42}-\angle S_{12}$. Circuit dimensions are in Table I. 
TABLE I

Circuit Parameters of the Two Fabricated Dual-Band RAt RACE COUPLERS $\left(\theta_{2}=5^{\circ} @ f=f_{1}\right)$

\begin{tabular}{|c|c|c|c|c|c|}
\hline$R$ & $\theta_{1}(\mathrm{deg})$ & $Z_{1}(\Omega)$ & $Z_{2}(\Omega)$ & $\theta_{S}(\mathrm{deg})$ & $Z_{S}(\Omega)$ \\
\hline 1 & 23.83 & 83.68 & 83.68 & 57.66 & 104.4 \\
\hline 4 & 15.25 & 143.5 & 35.88 & 64.52 & 111.8 \\
\hline
\end{tabular}

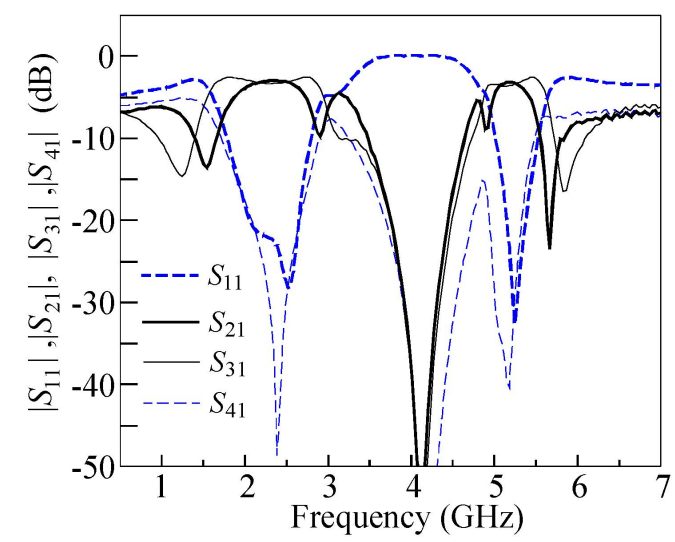

Fig. 5. Measured magnitude responses of the fabricated rat race coupler with $R=1$. Circuit dimensions are given in Table I.

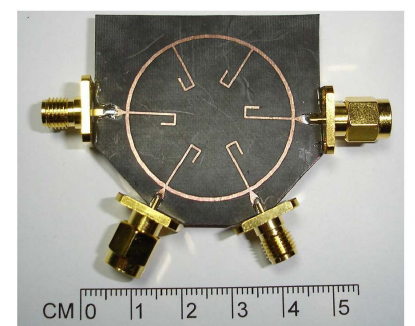

(a)

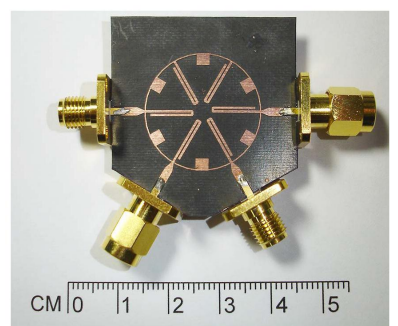

(b)
Fig. 6. Photos of the two fabricated circuits. (a) $R=1$. (b) $R=4$.

Fig. 6 shows the photos of the two fabricated circuits. In simulation, parasitic effects of the impedance junctions along the circumference in Fig. 6(b) offset the dips of $\left|S_{11}\right|$ and $\left|S_{41}\right|$ to $5.0 \mathrm{GHz}$ and $5.3 \mathrm{GHz}$, respectively. For compensation, the stub length is trimmed by $2^{\circ}$ and linewidth of the low- $Z$ is increased by $0.04 \mathrm{~mm}$.

\section{CONCLUSION}

Design technique for rat race ring coupler with dual-band operation together with circuit miniaturization is described. At the two designated frequencies, the circuit topology employs stepped-impedance line sections shunt with open stubs to play the role of each $\lambda / 4$-section in the conventional design. Formulation and design graphs for determining the circuit parameters are devised. It is found that the degree of circuit miniaturization is constrained by the linewidth resolution of the hi- $Z$ section of the fabrication process. The designed $2.45 / 5.2 \mathrm{GHz}$ ring hybrid with $R=4$ uses only $21 \%$ of the area of the conventional circuit. The stepped-impedance configuration introduces an extra power loss of $1 \%$ in the two bands. Dual-band ring hybrids with changed frequency ratios can also be designed in a similar fashion.

\section{ACKNOWLEDGEMENT}

This work was supported by the MoE ATU program and by the National Science Council, Taiwan, under Grants NSC 952221-E-009-037-MY2 and NSC 95-2752-E-009-003-PAE.

\section{REFERENCES}

[1] H. Hashemi and A. Hajimiri, "Concurrent multi-band low-noise amplifiers theory, design, and application," IEEE Trans. Microwave Theory Tech., vol. 50, no. 1, pp. 288-301, Jan. 2002.

[2] Y.-L. Kuo and K.-L. Wong, "Printed double-T monopole antenna for 2.4/5.2 GHz dual-band WLAN operations," IEEE Trans. Antennas Propagat., vol. 51, no.9, pp. 2187-2192, Sep. 2003.

[3] J.-T. Kuo, T.-H. Yeh and C.-C. Yeh, "Design of microstrip bandpass filters with a dual-passband response," IEEE Trans. Microwave Theory Tech., vol. 53, no. 4, pp. 1331-1337, Apr. 2005.

[4] S.-F. R. Chang, W.-L. Chen, S.-C. Chang, C.-K. Tu, C.-L. Wei, C.-H. Chieh, C.-H. Tsai, J. Chen and A. Chen, "A dual-band RF transceiver for multi-standard WLAN applications," IEEE Trans. Microwave Theory Tech., vol. 53, no. 3, pp. 1048-1055, Mar. 2005.

[5] I. H. Lin, C. Caloz, and T. Itoh, "A branch line coupler with two arbitrary operating frequencies using left handed transmission lines," in IEEE-S Int. Dig., pp. 325-328, Jun. 2003.

[6] K. M. Cheng and F.-L. Wong, "A novel approach to the design and implementation of dual-band compact planar $90^{\circ}$ branchline coupler," IEEE Trans. Microwave Theory Tech., vol. 52, no. 11, pp. 2458-2463, Nov. 2004.

[7] M.-J. Park and B. Lee, "Dual-band cross-coupled branch line coupler," IEEE Microwave Wireless Compon. Lett., vol. 15, no. 10, pp. 655-657, Oct. 2005.

[8] C. Collado, A. Grau, and F. De Flaviis, "Dual-band planar quardrature hybrid with enhanced bandwidth response," IEEE Trans. Microwave Theory Tech., vol. 54, no. 1, pp. 180-188, Jan. 2006.

[9] K. M. Cheng and F.-L. Wong, "A novel rat race coupler design for dual-band applications," IEEE Microwave Wireless Compon. Lett., vol. 15, no.8, pp. 521-523, Aug. 2005.

[10] R. K. Settaluri, G. Sundberg, A. Weisshaar, and V. K. Tripathi, "Compact folded line rat-race hybrid couplers," IEEE Microwave Guided Wave Lett., vol. 10, no. 2, pp. 61-63, Feb. 2000.

[11] H. Ghali and T. A. Moselhy, "Miniaturized fractal rat-race, branch-line, and coupled-line hybrids," IEEE Trans. Microwave Theory Tech., vol. 52, no. 11, pp. 2513-2520, Nov. 2004.

[12] J.-T. Kuo, J.-S. Wu and Y.-C. Chiou, "Miniaturized rat race coupler with suppression of spurious passband," IEEE Microwave Wireless Compon. Lett., vol. 17, no.1, pp. 46-48, Jan. 2007.

[13] Zeland Software Inc., IE3D Simulator, Jan., 2001. 